\title{
X-ray Cavities and Cooling Flows
}

\author{
Paul E. J. Nulsen \\ School of Engineering Physics, University of Wollongong, Wollongong \\ NSW 2522, Australia, and Harvard-Smithsonian Center for \\ Astrophysics, 60 Garden St, Cambridge, MA 02138, USA \\ Brian R. McNamara \\ Department of Physics and Astronomy, Ohio University, Athens, $\mathrm{OH}$ \\ 45\%01, USA \\ Laurence P. David \\ Harvard-Smithsonian Center for Astrophysics, 60 Garden St, \\ Cambridge, MA 02138, USA \\ Michael W. Wise \\ Massachusetts Institute of Technology, Center for Space Research, 70 \\ Vassar St, Building 37, Cambridge, MA 02139, USA
}

\begin{abstract}
Recent data have radically altered the X-ray perspective on cooling flow clusters. X-ray spectra show that very little of the hot intra-cluster medium is cooler than about $1 \mathrm{keV}$, despite having short cooling times. In an increasing number of cooling flow clusters, the lobes of a central radio source are found to have created cavities in the hot gas. Generally, the cavities are not overpressured relative to the intra-cluster gas, but act as buoyant bubbles of radio emitting plasma that drive circulation as they rise, mixing and heating the intra-cluster gas. All this points to the radio source, i.e., an active galactic nucleus, as the heat source that prevents gas from cooling to low temperatures. However, heating due to bubbles alone seems to be insufficient, so the energetics of cooling flows remain obscure. We briefly review the data and theory supporting this view and discuss the energetics of cooling flows.
\end{abstract}

\section{Introduction}

The radiative cooling time of the hot intergalactic gas close to the centers of about 70 percent of rich clusters of galaxies is significantly shorter than the Hubble time. These systems are known as cooling flows (Fabian 1994). Over the lifetime of a cooling flow cluster, radiative losses have a significant impact on the gas unless the radiated heat is replaced. Cooling gas is compressed in order to maintain hydrostatic equilibrium, causing inflow near to the cluster center and the deposition of large quantities of cool gas. X-ray data from Chandra and $X M M-N e w t o n$ confirm central cooling times as short as $10^{8}-10^{9} \mathrm{y}$ in many clusters (e.g. David et al. 2001), highlighting the significance of radiative losses. However, $100-1000 \mathrm{M}_{\odot} \mathrm{y}^{-1}$ of cold gas should be deposited by cooling flows 
(e.g. White, Jones \& Forman 1997) and very little evidence is found of this gas. Many forms of cool gas (e.g. Crawford et al. 1999; Edge 2001) and recent star formation (e.g. Mittaz et al. 2001) have been found at the centers of cooling flow clusters, but the amounts fall well short of those expected. Cooling flows also occur in groups and isolated elliptical galaxies (Mathews \& Brighenti 2003).

$\mathrm{X}$-ray spectra from the Reflection Grating Spectrograph (RGS) on $X M M$ Newton show that there is very little gas cooler than about $1 \mathrm{keV}$ in cluster cooling flows. If the late stages of cooling are isobaric as expected, then the luminosity in a line is $L_{\text {line }}=\dot{M}\left(5 k /\left(2 \mu m_{\mathrm{H}}\right) \int_{0}^{T_{\max }} \Lambda_{\text {line }}(T) / \Lambda(T) d T\right.$, where $\dot{M}$ is the deposition rate of cooled gas, $\Lambda$ is the cooling function and $\Lambda_{\text {line }}$ the part of the cooling function due to the line. This prediction is quite robust for low temperature lines, but RGS data show that some low energy lines are at least an order of magnitude weaker than expected (e.g. Peterson et al. 2003).

\section{Radio Lobe Cavities in Cooling Flows}

Burns (1990) found that 70 percent of $\mathrm{cD}$ galaxies in cooling flow clusters are radio loud, compared to 25 percent overall. Observations with Chandra reveal a growing list of clusters where radio lobes at the cluster center have created cavities in the hot intra-cluster gas. Some examples are Perseus (Böhringer et al. 1993; Fabian et al. 2000), Hydra A (McNamara et al. 2000), Abell 2052 (Blanton et al. 2001), RBS797 (Schindler et al. 2001), MKW3S (Mazzotta et al. 2002) and Abell 4059 (Heinz et al. 2002).

Contrary to expectation, there is little evidence of shocks driven by expanding radio lobes in most systems (but see Kraft et al. 2003; Fabian et al. 2003). Furthermore, the coolest $\mathrm{X}$-ray emitting gas surrounds the cavities in many systems (e.g. Nulsen et al. 2002). It would be very surprising to find the lowest entropy gas close to the origin of a strong shock. Lastly, the equipartition pressure of the radio lobes is typically about one tenth of the surrounding gas pressure. All this argues that the radio lobes are at nearly the same pressure as the surrounding gas.

The cool gas around the cavities is surprising. Nulsen et al. (2002) argued that this is not due to shock induced cooling or magneto-hydrodynamic shocks in Hydra A. However, in a temperature map, they found a plume of cool gas extending from the center to beyond the radio lobe cavities in Hydra A. This suggests that repeated radio outbursts have produced buoyant bubbles (cavities) that drive outflow along the radio axis, lifting some low entropy gas from the cluster center. Numerical simulations support this model (Brüggen et al. 2002).

\section{Energetics}

The question of what prevents gas from cooling to low temperatures in cooling flows remains a major issue. The heat required to make up for radiative losses from the region where the cooling time is shorter than the age of a cluster is typically $10^{44}-10^{45} \mathrm{erg} \mathrm{s}^{-1}$. Also, a significant amount of gas at the cluster center must be maintained with cooling times of $10^{8}-10^{9} \mathrm{y}$. This is very difficult to achieve without a process that involves feedback. 
Radio lobes are powered by an AGN, which is likely fueled by cooled and cooling gas. If the mechanical energy input due to the cavities is appreciable, this provides a feedback mechanism linking cooling and AGN heating. We consider the energy input by the bubbles, using Hydra $\mathrm{A}$ for illustration (David et al. 2001). The work of creating a bubble in local pressure equilibrium is $p V \simeq$ $2.8 \times 10^{59} \mathrm{erg}$ for the SW cavity of Hydra A. The free energy of a bubble is the sum of this and its thermal energy, giving the enthalpy $\gamma p V /(\gamma-1)$, where $\gamma$ is the ratio of specific heats. We double this again to allow for the NE bubble. If the cavity is dominated by relativistic plasma, then $\gamma=4 / 3$ and the total free energy of the two cavities in Hydra $\mathrm{A} \simeq 2.2 \times 10^{60} \mathrm{erg}$. If all of this is thermalized within the cooling flow region of Hydra A, then it can prevent gas from cooling for $2.3 \times 10^{8} \mathrm{y}$.

Churazov et al. (2002) argue that the enthalpy of a rising adiabatic bubble decreases with pressure, and the loss goes into heating the gas. A bubble rises as the ICM falls in around it, converting potential energy to kinetic energy, which is then dissipated in the bubble's wake. The potential energy released when a bubble of volume $V$ rises a distance $\delta R$ is $\delta E=\rho V g \delta R$, where $\rho$ is the density of the ICM and $g$ the acceleration due to gravity. From the equation of hydrostatic equilibrium, $\rho g=-d p / d r$, so that $\delta E=-V d p / d r \delta R=-V \delta p$. For an adiabatic bubble, $p V^{\gamma}=$ constant, and this result is readily integrated, giving the energy dissipated over a finite length of wake, $\Delta E=H_{0}-H$, where the enthalpy depends on the pressure through $H=H_{0}\left(p / p_{0}\right)^{(\gamma-1) / \gamma}$. For Hydra A, with $\gamma=4 / 3$, about half of the free energy is dissipated in the cooling flow region. If the bubble is non-adiabatic, more energy is deposited in the core.

While Hydra A is a very powerful FRI radio source, its cavities are not exceptional. Indeed, the existence of "ghost" cavities (e.g. McNamara et al. 2001) tells us that radio lifetime can be shorter than bubble lifetime and we should not expect a strong correlation between bubble energy and radio power. There may also be other energy inputs from an AGN, including direct injection of relativistic particles, uncollimated outflows, or Compton heating.

It is generally difficult to heat from the center of a cluster without creating a well mixed, isentropic core (Fabian et al. 2001; Brighenti \& Mathews 2003). Slow heating drives steady convection. Fast heating drives a shock, causing entropy inversion, then convection and mixing. Mixing is less thorough if energy is deposited off center, forming bubbles. However, to prevent the bulk of the lowest entropy gas from cooling to low temperatures, most gas must be heated substantially at some stage and so take part in large-scale convection, tending to disrupt observed abundance gradients (e.g. David et al. 2001).

Zakamska \& Narayan (2003) have shown that thermal conduction can balance radiative losses in some, but not all, cooling flows. However, since it involves no feedback, maintaining cool gas by thermal conduction requires very fine tuning (e.g. Bregman \& David 1988). Also, thermal conductivity must be suppressed to explain observed structures in several clusters. It has been argued that these are special cases, but this is harder to accept for the large scale suppression found by Markevitch et al. (2003).

Even when suppressed by a factor of 10 or more, thermal conduction can balance radiative losses in the outer parts of cluster cooling flows. Thus, thermal conduction may augment AGN heating in clusters. In that case, AGN heating 
need only account for radiative losses from near to the cluster center. Thermal conduction falls rapidly with temperature, and so is less likely to be significant in groups and isolated elliptical galaxies. On the other hand, their energetics are less demanding, since a single AGN outburst can disrupt the hot interstellar medium of an isolated elliptical (e.g. Finoguenov \& Jones 2001).

It has long been argued that major mergers can completely disrupt a cooling flow (McGlynn \& Fabian 1984) and a variety of mechanisms have been proposed to tap the energy of mergers to prevent gas from cooling (e.g. Motl et al. 2004). However, in minor mergers and infall, most energy is deposited in the outer parts of clusters. Stable stratification, and the huge density and pressure contrast from center to edge are obstacles to getting energy from the outer regions deposited in the cluster core. Furthermore, without feedback it is very difficult for such a process to maintain short cooling times in the cluster core. As for thermal conduction, these effects may augment AGN heating.

\section{Discussion and Conclusions}

There is good evidence of AGN heating in cooling flows, but it remains unclear whether it is significant for cooling flows as a whole. Since AGN heating is linked to cooling by feedback, this process can plausibly explain how gas can be maintained with short cooling times in cooling flows. AGN heating probably needs to be augmented in order to account for global energetics of cooling flows. Many details of the heating process remain obscure. In particular, it is unclear how a cluster can be heated from its center without producing a constant entropy core and mixing out observed abundance gradients.

To end on a speculative note, AGN outbursts also occur in isolated elliptical galaxies, where they can prevent the cooling of hot gas more readily than in clusters. If so, AGN feedback inhibits cooling, hence star formation, in almost any system dominated by hot gas. In that case, the effect of AGN feeback is imprinted on the galaxy luminosity function.

Acknowledgments. This work was partly supported by the Australian Research Council and by NASA grant NAS8-01130.

\section{References}

Blanton, E. L., Sarazin, C. L., McNamara, B. R., \& Wise, M. W., 2001, ApJ, 558, L15 Böhringer, H., Voges, W., Fabian, A. C., Edge, A. C., \& Neumann, D. M. 1993, MNRAS, 264, L25

Bregman, J. N., \& David, L. P. 1988, ApJ, 326, 639

Brighenti, F., \& Mathews, W. G. 2003, ApJ, 587, 580

Brüggen, M., Kaiser, C. R., Churazov, E., \& Enßlin, T. A. 2002, MNRAS, 331, 545

Burns, J. O. 1990, AJ, 99,14

Churazov, E., Sunyaev, R., Forman, W., \& Böhringer, H. 2002, MNRAS, 332, 729

Crawford, C. S., Allen, S. W., Ebeling, H., Edge, A. C., \& Fabian, A. C. 1999, MNRAS, 306,857

David, L. P., Nulsen, P. E. J., McNamara, B. R., Forman, W. R., Jones, C., Ponman, T., \& Robertson, B., Wise, M. 2001, ApJ, 557, 546 
Edge, A. C. 2001, MNRAS, 328, 762

Fabian, A. C. 1994, ARA\&A, 32, 277

Fabian, A. C. et al. 2000, MNRAS, 318, L65

Fabian, A. C., Mushotzky, R. F., Nulsen, P. E. J., Peterson, J. R. 2001, MNRAS, 321, $20 \mathrm{P}$

Fabian, A. C. et al. 2003, MNRAS, in press (astro-ph/0306036)

Finoguenov, A., \& Jones, C. 2001, ApJ, 547, L107

Heinz, S., Choi, Y.-Y., Reynolds, C. S., \& Begelman, M. C. 2002, ApJ, 569, L79

Kraft, R. P., Vázquez, S. E., Forman, W. R., Jones, C., Murray, S. S., Hardcastle, M. J., Worrall, D. M., \& Churazov, E. 2003, ApJ, 592, 129

Markevitch, M. et al. 2003, ApJ, 586, L19

Mathews, W. G., \& Brighenti, F. 2003, ARA\&A, 41, 191

Mazzotta, P., Kaastra, J. S., Paerels, F. B., Ferrigno, C., Colafrancesco, S., Mewe, R., \& Forman, W. R. 2002, ApJ, 567, L37

McGlynn, T. A., \& Fabian, A. C. 1984, MNRAS, 208, 709

McNamara, B. R. et al. 2000, ApJ, 534, L135

McNamara, B. R. et al. 2001, ApJ, 562, L149

Mittaz, J. P. D. et al. 2001, A\&A, 365, L98

Motl, P. M., Burns, J. O., Loken, C., Norman, M. L., \& Bryan, G. 2004, ApJ, 606, 635

Nulsen, P. E. J., David, L. P., McNamara, B. R., Jones, C., Forman, W. R., Wise, M. 2002, ApJ, 568, 163

Peterson, J. R., Kahn, S. M., Paerels, F. B. S., Kaastra, J. S., Tamura, T., Bleeker, J. A. M., Ferrigno, Jernigan, J. G. 2003, ApJ, 590, 207

Peres, C. B., Fabian, A. C., Edge, A. C., Allen, S. W., Johnstone, R. M., White, D. A. 1998, MNRAS, 298, 416

Schindler, S., Castillo-Morales, A., De Filippis, E., Schwope, A., Wambsganss, J. 2001, A\&A, 376, L27

White, D. A., Jones, C., Forman, W.R. 1997, MNRAS, 292, 419

Zakamska, N. L., \& Narayan, R. 2003, ApJ, 582, 162 